\title{
DATOS SOBRE LA DIETA DE TYTO ALBA \\ $Y$ BUBO BUBO EN ALICANTE \\ (SE DE IBERIA)
}

Por J. R. VERICAD $\left({ }^{*}\right)$, A. ESCARRE $(* *)$ y E. RODRIGUEZ

S U M ARIO

Página

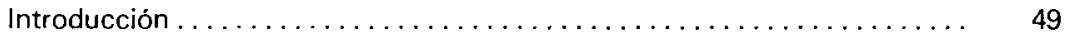

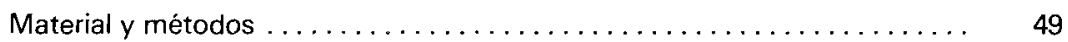

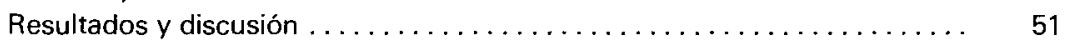

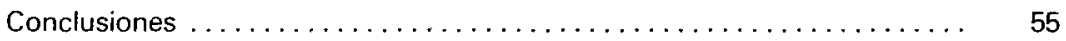

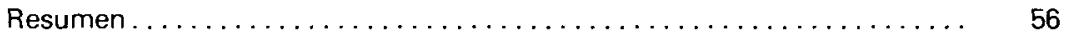

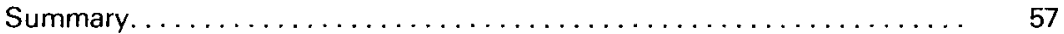

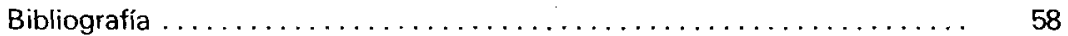

* Centro Pirenaico de Biología Experimental, Jaca (Huesca).

* Departamento de Biología y Geología, C.E.U., Alicante.

(Dirección actual: Departamento de Ecología, Facultad de Biologia, Barcelona.) 

Las dos revisiones más recientes sobre la alimentación de $T$. alba en Europa se deben a SCHMIDT (1973) y HERRERA (1974). En España hasta el momento se han publicado algunos trabajos sobre el tema, pero de preferencia enfocados hacia la faunística de los micromamíferos depredados. Así, los más recientes son para Cataluña, NADAL y PALAUS (1967) y SANS-COMA (1970, 1974a, 1974b), para la región cantábrica, HEIM DE BALSAC y BEAUFORT (1960), para Asturias BRAÑA (1974) y para Andalucía occidental VALVERDE (1967) y HERRERA (1973).

Sobre la alimentación de $B$. bubo, la publicación de JANOSSY y SCHMIDT (1970) representa una notable puesta al dia de la misma y su variación dentro del paleártico.En España, PEREZ-CHISCANO (1969, 1973) y ARAUJO (1973) dan a conocer algunos datos sobre la alimentación de esta especie en Extremadura, mientras que RUIZ y CAMACHO (1973) lo hacen sobre la alimentación de $B$. bubo en Sierra Nevada.

Desde el punto de vista de la dieta de estas dos rapaces, el SE de Iberia no es nada conocido por lo que hemos creído interesante darla a conocer.

MATERIAL Y METODOS

Se han analizado egagrópilas de $T$. alba de quince localidades de la provincia de Alicante (Fig. 1) totalizando las presas vertebradas identificadas 6.570 ejemplares. De $B$. bubo se han recolectado unas pocas regurgitaciones correspondientes a cuatro localidades (Fig. 1) de la prot vincia dando un total de 52 presas vertebradas de seis especies.

Para la determinación del peso seco de cada especie de presa se ha calculado el valor medio de cada una desecando totalmente de tres a cinco ejemplares de tamaño medio en una estufa entre 110 y $115^{\circ} \mathrm{C}$.

Los autores son conscientes del valor limitado de las conclusiones por varias razones; no se sabe hasta qué punto son representativos los datos de cada localidad por ser escasos o por no conocer en qué época 


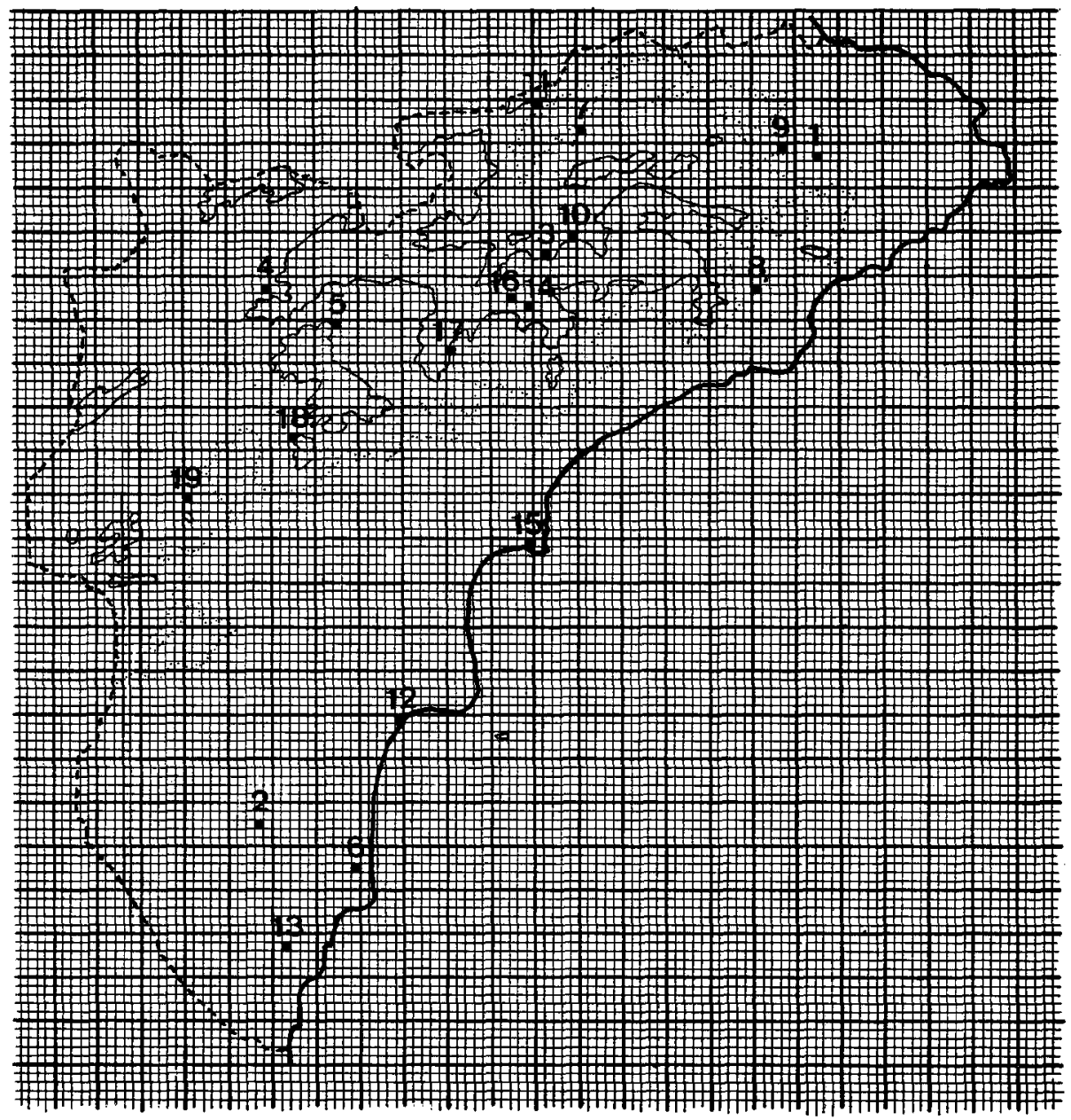

FIGURA 1. - Mapa de la provincia de Alicante donde se señalan en cuadrículas UTM de $1 \times 1 \mathrm{~km}$. los lugares de recogida de egagrópilas de Tyto alba 11: Alcalali; 2: Algorfa; 3: Benifallim; 4: Biar; 5: Castalla; 6: Dolores; 7: Gayanes; 8: La Nucia; 9: Murla; 10: Penáguila; 11: Planes; 12: Salinas de Santa Pola; 13: San Miguel de Salinas; 14: Torremanzanas, y 15: Vistahermosa) y Bubo bubo (16: Torremanzanas; 17: Peñas de Roset (Jijona); 18: La Silla del Cid, y 19: Monóvar).

Se indican las curvas de nivel de $400 \mathrm{~m}$. (linea punteada) y 800 m.s.m. (linea continua). 
fueron producidos los restos estudiados. Por otro lado no es muy claro hasta qué punto es lícito el considerar conjuntamente todas las localidades.

\section{RESULTADO Y DISCUSION}

Sobre la dieta de la lechuza a la vista de la tabla I se observa que las presas básicas en cuanto a presencia en las muestras estudiadas son Mus musculus, presente en todas las localidades, seguido por las aves, Suncus struscus, Crocidura russula, Apodemus sylvaticus y Rattus rattus presentes en más del 86 por ciento de las muestras. Las presas restantes se presentan de un 13 por ciento (Oryctolagus cuniculus) al 66 por ciento (Eliomys quercinus) de las localidades. Los artrópodos, representados por los insectos, carecen totalmente de importancia, pues sólo se han encontrado rastros de ellos difícilmente cuantificables, y se presentan sólo en menos de la mitad de las muestras mientras que se presentan en algo más del 78 por ciento de las muestras en España sudoccidental (HERRERA, 1973).

Considerando las presas vertebradas en tres grandes grupos resulta que para el conjunto de las mismas y de las muestras tanto respecto al número como a la biomasa de peso seco los mamíferos superan el 84 y el 79 por ciento respectivamente, y las aves alcanzan el 15,6 y 20,9 por ciento. Los reptiles carecen de importancia en la dieta de $T$. alba 10,2 y 0,0 por ciento).

Reptiles: Como acabamos de indicar tanto en su aportación numérica como de biomasa de peso seco a la dieta de la lechuza es insignificante. Las especies depredadas son saurios que por orden de importancia en cuanto a aportación numérica son Lacerta hispanica, Tarentola mauritanica, Psammodromus sp. y Hemidactylus turcicus. Es interesante destacar que aunque varias referencias anteriores señalan a los lacértidos como presa de la lechuza en el sur de Europa (GUERIN, 1928; WITTE, 1964; VALVERDE, 1967; HERRERA, 1973), los gecónidos como presa de la lechuza han sido citados sólo por NOS (1960) y MARTINEZRICA (1974), indicando este último autor que los restos de las dos especies de salamanquesa ocupaban cerca de la mitad del volumen de la egagrópilas recogidas por él en el sureste de Mallorca. Aparte de nuestras localidades, donde aparecen gecónidos junto a lacértidos como presa, otros lotes de egagrópilas de localidades levantinas (prov. de $\mathrm{Va}$ - 


\begin{tabular}{|c|c|c|c|c|c|c|c|c|c|c|c|c|c|c|c|c|}
\hline & 1 & 2 & 3 & 4 & 5 & 6 & 7 & 8 & 9 & 10 & 11 & 12 & 13 & 14 & 15 & 16 \\
\hline Insecta & + & - & + & - & + & - & + & - & + & + & - & - & - & - & - & - \\
\hline Reptilia & $\begin{array}{l}1 \\
2.1\end{array}$ & - & $\begin{array}{l}0.6 \\
0.1\end{array}$ & $\overline{-}$ & - & $\begin{array}{l}0.3 \\
0.1\end{array}$ & $\begin{array}{l}- \\
-\end{array}$ & - & $\begin{array}{l}1.1^{\prime} \\
0.3\end{array}$ & - & $\begin{array}{l}- \\
-\end{array}$ & - & - & - & - & $\begin{array}{l}0.2 \\
0.0\end{array}$ \\
\hline Aves & $\begin{array}{l}28.3 \\
36.4\end{array}$ & $\begin{array}{l}33.8 \\
35.6\end{array}$ & $\begin{array}{r}9.1 \\
12.5\end{array}$ & $\begin{array}{r}11.5 \\
13.1\end{array}$ & $\begin{array}{l}24.9 \\
31.3\end{array}$ & - & $\begin{array}{l}2.7 \\
4.6\end{array}$ & $\begin{array}{l}17.0 \\
22.1\end{array}$ & $\begin{array}{l}6.0 \\
9.3\end{array}$ & $\begin{array}{l}13.2 \\
19.8\end{array}$ & $\begin{array}{r}9.0 \\
14.2\end{array}$ & $\begin{array}{l}5.7 \\
7.5\end{array}$ & $\begin{array}{l}4.2 \\
4.9\end{array}$ & $\begin{array}{l}1.2 \\
2.1\end{array}$ & $\begin{array}{l}41.5 \\
47.6\end{array}$ & $\begin{array}{l}15.6 \\
20.9\end{array}$ \\
\hline $\begin{array}{l}\text { Suncus } \\
\text { etruscus }\end{array}$ & $\begin{array}{l}1.4 \\
0.1\end{array}$ & $\begin{array}{l}0.5 \\
0.0\end{array}$ & $\begin{array}{l}1.8 \\
0.1\end{array}$ & $\begin{array}{r}11.5 \\
0.7\end{array}$ & $\begin{array}{l}1.1 \\
0.1\end{array}$ & $\begin{array}{l}3.6 \\
0.3\end{array}$ & $\begin{array}{l}1.2 \\
0.1\end{array}$ & $\begin{array}{l}1.7 \\
0.1\end{array}$ & $\begin{array}{l}2.2 \\
0.2\end{array}$ & $\begin{array}{l}1.0 \\
0.1\end{array}$ & $\begin{array}{l}3.2 \\
0.3\end{array}$ & $\begin{array}{l}1.1 \\
0.1\end{array}$ & $\begin{array}{l}0.3 \\
0.0\end{array}$ & $\begin{array}{l}1.5 \\
0.2\end{array}$ & - & $\begin{array}{l}1.4 \\
0.1\end{array}$ \\
\hline $\begin{array}{l}\text { Crocidura } \\
\text { russula }\end{array}$ & $\begin{array}{r}25.6 \\
8.1\end{array}$ & $\begin{array}{r}37.3 \\
9.6\end{array}$ & $\begin{array}{l}38.7 \\
12.9\end{array}$ & $\begin{array}{l}7.7 \\
2.1\end{array}$ & $\begin{array}{r}16.3 \\
5.0\end{array}$ & $\begin{array}{l}28.7 \\
11.0\end{array}$ & $\begin{array}{l}26.2 \\
10.6\end{array}$ & $\begin{array}{l}47.8 \\
15.2\end{array}$ & $\begin{array}{l}44.8 \\
17.0\end{array}$ & $\begin{array}{l}32.6 \\
11.4\end{array}$ & $\begin{array}{l}34.2 \\
13.2\end{array}$ & $\begin{array}{r}19.9 \\
6.4\end{array}$ & $\begin{array}{l}1: 6 \\
0.5\end{array}$ & $\begin{array}{l}46.1 \\
19.4\end{array}$ & - & $\begin{array}{r}27.2 \\
8.9\end{array}$ \\
\hline Chiroptera & - & - & $\begin{array}{l}0.6 \\
0.6\end{array}$ & $\begin{array}{l}3.8 \\
3.6\end{array}$ & $\begin{array}{l}0.2 \\
0.2\end{array}$ & $\begin{array}{l}0.9 \\
1.2\end{array}$ & - & $\begin{array}{l}0.5 \\
0.6\end{array}$ & - & $\begin{array}{l}0.1 \\
0.2\end{array}$ & - & $\begin{array}{l}- \\
-\end{array}$ & - & $\begin{array}{l}0.9 \\
1.3\end{array}$ & - & $\begin{array}{l}0.2 \\
0.3\end{array}$ \\
\hline $\begin{array}{l}\text { Apodemus } \\
\text { sylvaticus }\end{array}$ & $\begin{array}{l}18.0 \\
24.7\end{array}$ & - & $\begin{array}{l}33.5 \\
48.6\end{array}$ & $\begin{array}{l}7.7 \\
9.3\end{array}$ & $\begin{array}{r}8.5 \\
11.3\end{array}$ & $\begin{array}{l}1.5 \\
2.5\end{array}$ & $\begin{array}{r}6.7 \\
11.8\end{array}$ & $\begin{array}{l}14.8 \\
20.4\end{array}$ & $\begin{array}{l}20.8 \\
34.2\end{array}$ & $\begin{array}{l}23.2 \\
35.3\end{array}$ & $\begin{array}{r}6.4 \\
10.8\end{array}$ & $\begin{array}{l}2.3 \\
3.2\end{array}$ & $\begin{array}{l}1.2 \\
1.5\end{array}$ & $\begin{array}{l}26.5 \\
48.2\end{array}$ & $\begin{array}{l}3.0 \\
3.7\end{array}$ & $\begin{array}{l}12.9 \\
18.4\end{array}$ \\
\hline $\begin{array}{l}\text { Rattus } \\
\text { rattus }\end{array}$ & $\begin{array}{l}1.2 \\
7.3\end{array}$ & $\begin{array}{c}8 \\
40.6\end{array}$ & $\begin{array}{l}0.9 \\
6.0\end{array}$ & $\begin{array}{r}3.8 \\
21.2\end{array}$ & $11^{1.8}$ & $\begin{array}{r}1.5 \\
11.4\end{array}$ & - & $\begin{array}{r}4.6 \\
28.9\end{array}$ & $\begin{array}{l}1.1 \\
8.2\end{array}$ & $\begin{array}{l}1.0 \\
7.1\end{array}$ & $\begin{array}{l}1.3 \\
9.9\end{array}$ & $\begin{array}{r}2.7 \\
17.0\end{array}$ & $\begin{array}{r}2.7 \\
15.4\end{array}$ & - & $\begin{array}{l}1.5 \\
8.6\end{array}$ & $\begin{array}{c}2 \\
12.9\end{array}$ \\
\hline $\begin{array}{l}\text { Mus } \\
\text { musculus }\end{array}$ & $\begin{array}{l}24.1 \\
20\end{array}$ & $\begin{array}{l}19.9 \\
13.6\end{array}$ & $\begin{array}{l}13.1 \\
11.6\end{array}$ & $\begin{array}{l}50 \\
36.8\end{array}$ & $\begin{array}{l}46.4 \\
37.7\end{array}$ & $\begin{array}{l}61.7 \\
62.7\end{array}$ & $\begin{array}{l}62.5 \\
67.1\end{array}$ & $\begin{array}{l}13.2 \\
11.1\end{array}$ & $\begin{array}{l}22.4 \\
22.5\end{array}$ & $\begin{array}{l}28.1 \\
26.1\end{array}$ & $\begin{array}{l}44.5 \\
45.5\end{array}$ & $\begin{array}{l}66.3 \\
56.1\end{array}$ & $\begin{array}{l}81.9 \\
62.8\end{array}$ & $\begin{array}{l}23.1 \\
25.6\end{array}$ & $\begin{array}{l}53.8 \\
39.9\end{array}$ & $\begin{array}{l}38.9 \\
33.7\end{array}$ \\
\hline $\begin{array}{l}\text { Eliomys } \\
\text { quercinus }\end{array}$ & $\begin{array}{c}0.3 \\
1.1\end{array}$ & - & $\begin{array}{l}1.8 \\
7.4\end{array}$ & $\begin{array}{r}3.8 \\
12.9\end{array}$ & $\begin{array}{l}0.3 \\
1.2\end{array}$ & $\begin{array}{l}0.3 \\
1.4\end{array}$ & - & $\begin{array}{l}0.4 \\
1.5\end{array}$ & $\begin{array}{l}1.1 \\
5.0\end{array}$ & $\begin{array}{l}- \\
-\end{array}$ & $\begin{array}{l}1.3 \\
6.0\end{array}$ & - & $\begin{array}{l}2.4 \\
8.3\end{array}$ & $\begin{array}{l}0.6 \\
3.1\end{array}$ & $\overline{-}$ & $\begin{array}{l}0.6 \\
2.4\end{array}$ \\
\hline $\begin{array}{l}\text { Arvicola } \\
\text { sapidus }\end{array}$ & - & - & - & - & $\begin{array}{l}0.4 \\
2\end{array}$ & $\begin{array}{l}0.9 \\
5.4\end{array}$ & $\begin{array}{l}0.3 \\
1.9\end{array}$ & - & $\begin{array}{l}0.5 \\
3.2\end{array}$ & $\begin{array}{l}- \\
-\end{array}$ & - & $\begin{array}{l}1.9 \\
9.6\end{array}$ & - & $\begin{array}{l}- \\
-\end{array}$ & - & $\begin{array}{l}0.2 \\
1.2\end{array}$ \\
\hline $\begin{array}{l}\text { Pitymys } \\
\text { duodecimcostatus }\end{array}$ & - & $\begin{array}{l}0.5 \\
0.5\end{array}$ & - & - & - & $\begin{array}{l}0.3 \\
0.4\end{array}$ & - & - & - & $\begin{array}{l}- \\
-\end{array}$ & - & - & $\begin{array}{l}5.7 \\
6.5\end{array}$ & - & - & $\begin{array}{l}0.6 \\
0.8\end{array}$ \\
\hline $\begin{array}{l}\text { Oryctolagus } \\
\text { cuniculus }\end{array}$ & - & - & - & - & - & $\begin{array}{l}0.3 \\
3.5\end{array}$ & $\begin{array}{l}0.3 \\
3.8\end{array}$ & - & - & - & - & - & - & - & - & $\begin{array}{l}0.0 \\
0.3\end{array}$ \\
\hline $\begin{array}{l}\text { Total individuos } \\
\text { Total gramos } \\
\text { peso seco }\end{array}$ & $\begin{array}{l}1.031 \\
3.956 .5\end{array}$ & $\begin{array}{l}201 \\
943.8\end{array}$ & $\begin{array}{c}329 \\
1.188 .3\end{array}$ & $\begin{array}{c}26 \\
112.9\end{array}$ & \begin{tabular}{|l|}
1.212 \\
4.768 .2
\end{tabular} & $\begin{array}{c}334 \\
1.051 .5\end{array}$ & $\begin{array}{l}328 \\
977\end{array}$ & $\begin{array}{c}764 \\
2.904 .4\end{array}$ & $\begin{array}{r}183 \\
583.1\end{array}$ & $\begin{array}{c}686 \\
2.366 .9\end{array}$ & $\begin{array}{r}155 \\
485.3\end{array}$ & $\begin{array}{r}261 \\
986.0\end{array}$ & $\begin{array}{c}670 \\
2.799 .0\end{array}$ & $\begin{array}{r}325 \\
935.8\end{array}$ & $\begin{array}{r}65 \\
280.4\end{array}$ & $\begin{array}{c}6.570 \\
24.262 .3\end{array}$ \\
\hline
\end{tabular}

TABLA I. - Espectro trófico de Tyto alba en la provincia de Alicante según las muestras estudiadas.

Las columnas 1 a 15 son las correspondientes a cada localidad (Fig. 1) en las que para cada tipo de presa se dan los porcentajes correspondientes a número (arriba) y biomasa de peso seco (abajo). En la columna 16 se dan los mismos porcentajes, pero para el conjunto de todas las localidades. En la base de cada columna se indica el número total de presas vertebradas (arriba) y la biomasa total en gramos de peso seco (abajo) para cada localidad (1 a 15) y para el conjunto de los mismos (base columna 16). 
lencia) han confirmado la depredación que sufren las dos salamanquesas por parte de la lechuza.

Aves: Los porcentajes respecto al número total de presas en cada localidad (Tabla 1) varían del 1,2 al 41,5 por ciento, siendo en todos los casos el gorrión común (Passer domesticus) el ave más solicitada, aspecto que coincide con los datos de HERRERA (1973), donde el gorrión común constituye el 54 por ciento de las aves depredadas, y de SCHMIDT (1973) para varios países de Europa. Considerando el conjunto de las muestras y de las presas vertebradas a las aves les corresponde en Alicante un porcentaje de 15,6, mientras que para España sudoccidental y Cataluña les corresponde sólo el 4,5 y el 2,4 por ciento (HERRERA, 1973, y SANS-COMA, 1974b).

Mamiferos: Sólo para tres de las quince muestras la proporción de insectívoros supera a la de los roedores. Considerando conjuntamente todas las localidades sobre el total de presas mamíferas corresponde el 34 por ciento a los insectívoros y el 65,6 por ciento a los roedores.

Los porcentajes numéricos de $C$. russula son claramente superiores a los de $S$. etruscus excepto en la localidad número 4 debido seguramente a la pequeñez de la muestra (Tabla I). En las dos especies es mayor la importancia numérica que la energética, tanto en cada localidad como para el conjunto de las mismas como queda reflejado en la columna 16 de la dicha tabla I, 1,4 y 0,1 por ciento para $S$. etruscus y 27,2 y 8,9 por ciento para $C$. russula. Respecto al porcentaje numérico de presencia de $S$. etruscus resulta ser bastante menor que los dados por HERRERA (1973) para España suroccidental y por KAHMANN y BROTZLER (1956) para Córcega, acercándose más a los valores conocidos para Cataluña (NADAL y PALAUS, 1967; SANS-COMA, 1970) y Huesca (VERICAD, 1971).

La contribùción de los quirópteros a la dieta de la lechuza en la región considerada, tal como se puede observar en la tabla I es mínima aunque se presentan en siete de las quince muestras. Las especies depredadas son por orden de importancia numérica Pipistrellus pipistrellus, Rhinolophus ferrum-equinum, Pipistrellus sp., Myotis myotis, Miniopterus schreibersi y Eptesicus serotinus.

De las seis especies de roedores implicadas resalta en la columna dieciséis de la tabla I la importancia que respecto a su porcentaje numérico tienen Mus musculus (38,9 por ciento) y Apodemus sy/vaticus 112,9 por ciento) y también respecto a la biomasa de peso seco aportada que 
entre las dos especies supera el cincuenta por ciento del conjunto de las presas vertebradas. Rattus rattus si bien tiene poca importancia numérica global (dos por ciento), su aportación energética es bastante mayor $(12,9$ por ciento), al contrario de $C$. russula. Eliomys quercinus no es una especie rara en la dieta de la lechuza pues se presenta en diez de las quince muestras, pero tanto su aportación numérica como en biomasa es baja $(0,6$ y 2,4 por ciento respectivamente). Algo similar podría decirse de Arvicola sapidus que, sin embargo, se presenta sólo en un tercio de las muestras. Pitymys duodecimcostatus se presenta sólo en tres de las localidades estudiadas y en porcentajes muy bajos respecto al total de las presas, excepto en la localidad número trece donde alcanza un porcentaje numérico de 5,7 .

Como se ve de la misma tabla I el conejo constituye para la lechuza una presa ocasional, tratándose en estos casos de ejemplares juveniles los depredados.

Sobre el espectro trófico de Bubo bubo en la provincia de Alicante resalta en la tabla II la importancia del conejo (O. cuniculus) y los erinaceidos entre las presas mamíferas. En comparación con los escasos datos españoles publicados, ya citados en la introducción, el búho real parece tener una alimentación menos ecléctica. Haría falta, sin embargo, series más largas de egagrópilas en la zona en estudio.

\section{CONCLUSIONES}

En el conjunto de las muestras estudiadas se puede considerar respecto a su importancia numérica como presas habituales predominantes a las aves, Crocidura russula, Apodemus sylvaticus y Mus musculus, como presas habituales no predominantes a Suncus etruscus y Rattus rattus y como presas esporádicas a reptiles saurios, quirópteros, Eliomys quercinus, Arvicola sapidus, Pitymys duodecimcostatus y Oryctolagus cuniculus. Los insectos, a tenor de lo visto, son presa esporádica de importancia numérica inapreciable respecto al total de presas. Al considerar la biomasa en gramos de peso aportada por cada presa, Rattus rattus pasaría a ser presa habitual predominante, mientras que Crocidura russula sería presa habitual no predominante.

Es de resaltar la escasa importancia del topillo Pitymys duodecimcostatus como presa de la lechuza por un lado y por otro el impacto que tienen las aves en la dieta de la lechuza en la zona considerada, en 
TABLA II. - Espectro trófico de Bubo bubo en Alicante según las muestras estudiadas.

\begin{tabular}{|c|c|c|c|c|c|c|c|}
\hline & & 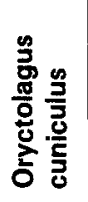 & 象 $\frac{\frac{\infty}{5}}{5}$ & 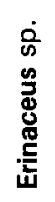 & 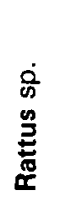 & $\stackrel{\infty}{\&}$ & 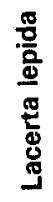 \\
\hline $\begin{array}{l}\text { Torremanzanas } \\
\text { Peñas de Roset } \\
\text { (Jijona) }\end{array}$ & YH27 & 5 & - & - & - & - & 1 \\
\hline $\begin{array}{l}\text { La Silla del Cid } \\
\text { Finca La Zafra } \\
\text { (Monóvar) }\end{array}$ & $\begin{array}{l}\text { XH9761 } \\
\text { XH8554 }\end{array}$ & 24 & - & 8 & - & - & - \\
\hline
\end{tabular}

contraste con los datos ya publicados sobre la alimentación de la lechuza.

El búho real se revela como depredador predominante de mamíferos según se deduce del escaso material estudiado.

RESUMEN

Este trabajo trata de la alimentación de la lechuza común ( $T$. alba) en quince localidades de Alicante (S. E. Iberia). El número total de presas vertebradas estudiadas es de 6.570. Las aves paseriformes presentan más importancia por término medio en su dieta que en otros lugares de España. Por el contrario el topillo $P$. duodecimcostatus, presa común en 
otros lugares de lberia, casi no tiene importancia en las localidades estudiadas.

Además se han analizado algunas regurgitaciones de búho real ( $B$. bubol, siendo el conejo y el erizo las presas básicas.

\section{SUMMARY}

This paper deals with the feeding habits of the Barn owl (T. alba) in 15 localities of Alicante (S. E. Spain). The amount of the studied vertebrate preys goes up to 6.570 . Passerine birds are, on the average, more important as a prey than elsewhere in Spain. Otherwise, the vole P. duodecimcostatus, commom prey elsewhere in Iberia, is very rarely concerned here.

Some pellets of Eagle owl ( $B$. bubo) have been also analyzed, being rabbit and hedgehog the ground preys. 
ARAUJO, J., 1973. - Algunas presas interesantes de Strigiformes y Accipitriformes. Ardeola, 19 (1): 24-25.

BRAÑA, F., 1974. - Estudio del régimen alimenticio de la lechuza común, Tyto alba (Scop.) en Asturias. Asturnatura, 2: 75-83.

GUERIN, G., 1928. - Regime et croissance de I'Effraye commune en Vendée. Lechevalier, París.

HEIM DE BALSAC, H., y F. BEAUFORT, 1969. - Contribution à l'étude des micromammifères du Nord-Ouest de I'Espagne (Santander, Asturias, Galice, León). Mammalia, 33: 630-658.

HERRERA, C. M., 1973. - Régimen alimenticio de Tyto alba en España suroccidental. Ardeola, 19 (2): 359-394.

HERRERA, C. M., 1974. - Tropic diversity of the Barn Owl Tyto alba in continental Western Europe. Ornis Scandinavica, 5 (2): 181-191.

JANOSSY, D., y E. SCHMIDT, 1970. - Die Nahrung des Uhus (Bubo bubo). Regionale und erdzeirliche Anderungen. Bonn. Zool. Beitr., 21 (1-2): 25-51.

KAHMANN, H., y A. BROTZLER, 1956. - Die Ernährung der Schleiereule (Tyto alba) und das Bild der Verbreitung Kleiner Säugetiere anf der Insel Korsika. Biol. Zbl. Leipzig, 76: 67-83.

MARTINEZ-RICA, J. P., 1974. - Contribución al estudio de la biología de los gecónidos ibéricos (Rept., Sauria). P. Cent. pir. Biol. exp., 5: 8-291.

NADAL, J., y X. PALAUS, 1967. - Micromamiferos hallados en egagrópilas de Tyto alba. P. Inst. Biol. Apl., 42: 5-15.

NOS, R., 1960. - Estudio de los ovillos regurgitados por una pareja de Tyto alba en la comarca de la Maresma (prov. Barcelona). Misc. Zool., 1: 3-10.

PEREZ-CHISCANO, J. P., 1969. - Estudio de una comunidad de aves de presa (primavera-verano 1968). Ardeola, 13 (2): 177-189.

PEREZ-CHISCANO, J. P., 1973. - Sumario informe sobre la alimentación de rapaces en el noreste de la provincia de Badajoz. Ardeola, 19: 331-336.

RUIZ, A., e I. CAMACHO, 1973. - Datos sobre la alimentación de Bubo bubo y determinación de micromamíferos en Sierra Nevada mediante egagrópilas. Cuad. C. Biol., 2 (2): 57-61. 
SANS-COMA, V., 1970.-Sobre la distribución-de micromamiferos del N.E. de la Peninsula lbérica, con algunas consideraciones metodológicas. P. Inst. Biol. Apl., 48: 125-144.

SANS-COMA, V., 1974a. - Contribución al conocimiento de los micromamíferos del nordeste de la Península lbérica y su interés biológico. Insectívoros y roedores en las egagrópilas de Tyto alba. Tesis Doctoral. Univ. Barcelona (sin publicar).

SANS-COMA, V., 1974b. - Sobre la alimentación de Tyto alba en la región continental catalana. Misc. Zool., 3 (4): 163-169.

SCHMIDT, E., 1973. - Die Nahrung der Schleiereule (Tyto alba) in Europa. Z. f., Angewandte Zoologie, 60 (1): 43-70.

VALVERDE, J. A., 1967. - Estructura de una comunidad de vertebrados terrestres. C. S. I. C.

VERICAD, J.-R., 1971.-Suncus etruscus y Microtus cabrerae en el Pirineo oscense. Pirineos, 101: 31-33.

WITTE, G., 1964. - Zur Systematik der Insektenfresser des Monte-Gargano-Gebietes (Italien). Boon. zool. Beitr, 15: 1-35. 